\title{
Sterilization of air filters for high pre-vacuum autoclaves $^{1}$
}

\author{
R. J. FALLON \\ From the Pathology Department, Ruchill Hospital, Glasgow
}

SYNOPSIS Experiments are described with glass fibre filter paper and complete glass filters as fitted to high pre-vacuum autoclaves to determine whether organisms can grow or penetrate through the filters. A filter consisting of four layers of glass fibre paper fitted inside a sterilizer chamber for a year and used for 96 sterilization cycles was found to have retained its initial efficiency.

Many high pre-vacuum sterilizers are now fitted with glass fibre filters. These are of much greater efficiency than the types of filter previously in use (M.R.C. Report, 1960). A glass fibre filter should be changed once every 12 months although this recommendation is to some extent arbitrary as many filters would probably give satisfactory service for a longer period. In places where there is much atmospheric pollution a coarse pre-filter will extend the life of the absolute filter.

Although these filters are highly efficient in removing bacteria from the air which passes through them, the possibility exists that micro-organisms might grow through them as has been found with other types of bacterial filter.

Experiments were carried out both with an intact filter and with the glass fibre filter medium which consisted of glass fibre felted into sheet-form of paper thickness. ${ }^{2}$ The outside of the filter was soaked with a broth culture of organisms, on one occasion Pseudomonas pyocyanea and on other occasions Chromobacterium prodigiosum (Serratia marcesens), and was incubated (in a closed container in order to keep the filter moist) either at room temperature or at $37^{\circ} \mathrm{C}$. for a week on each occasion. Despite the fact that it was not designed to withstand autoclaving, the filter was autoclaved in between experiments and even though it was re-inoculated with organisms as often as once a day in the final experiments, nutrient broth washings taken from the inside of the filter did not grow the organism used as a challenge. Cultures taken at the same time

'A report to the Medical Research Council working party on pressure-
steam sterilizers.

${ }^{2}$ Kindly supplied by Messrs. E. Mackley \& Co., Hawks Road, Gateshead, 8, Co. Durham.

Received for publication 17 November 1962. from the outside of the filter were positive. Chr. prodigiosum also failed to grow through squares of glass fibre paper placed on nutrient agar and left for a week at room temperature.

An intact filter was not challenged with fungi but filter paper squares were when $10 \mathrm{~cm}$. squares of glass fibre paper were placed on Sabouraud's dextrose agar in $9 \mathrm{~cm}$. petri dishes. They were inoculated in the centre with a fungus (atypical Alternaria sp.) isolated from the laboratory air. At the end of a week, by which time the mycelium nearly reached the edge of the square, the paper was removed. On each occasion the mould penetrated the paper as evidenced by growth over a small circular area on the centre of the medium.

Although the conditions of the latter experiment were artificial in that the filter material was in contact with a nutrient medium it seems possible that in a warm and humid stiuation moulds might, in time, grow through a filter. (It is, however, important to note that an intact filter may have more than one layer of glass fibre paper.) This danger could be avoided if the filter was sterilized on each occasion before it was used. Such a system is already used for both ceramic and metal-wool filters fitted to sterilisers.

A filter ${ }^{3}$ consisting of four layers of glass fibre paper held in a stainless steel canister was placed inside a $4.2 \mathrm{cu}$. $\mathrm{ft}$. high pre-vacuum experimental sterilizer which has been described elsewhere (Fallon, 1961). The filter was placed on the inner end of the air inlet to the chamber. It was left there for a year, the sterilizer being used for 96 sterilization cycles during this time. Most cycles were high prevacuum using steam at temperatures of $121^{\circ} \mathrm{C}$., ${ }^{3}$ Kindly supplied by Messrs. Vokes Ltd., Henley Park, Guildford,
Surrey. 
TABLE

Filter Test

Air Filter Performance

New Filter

Before Sterilization

After Sterilization

${ }^{1}$ Methylene blue penetration ( $\%$ )

${ }^{1}$ Sodium chloride penetration $(\%)$

Monodispersed spore penetration (\%)

0.002

1 These tests are comparable.

$126^{\circ} \mathrm{C}$, , or $134^{\circ} \mathrm{C}$. but some were gravity displacement cycles. The filter was thus subjected to a wide range of conditions. At the end of this time the filter was forwarded for testing to Surg. Cmdr. H. M. Darlow at the Microbiological Research Establishment of the War Office. The results of the initial and final tests on the filter, together with one on a new filter tested at the same time, are given in the table and show that the filter withstood the test without deterioration.

Before installing glass fibre air filters inside high pre-vacuum sterilizers as a routine practice, a prolonged test should be carried out in a commercial model of sterilizer in daily use. Only then can the above findings be assessed finally but there can be little doubt that in view of the nature of these findings it should be possible for a suitable filter, designed with the collaboration of both filter suppliers and sterilizer manufacturers, to withstand repeated sterilization cycles.

I wish to acknowledge the kind help of Dr. Darlow in testing several filters during the course of these experiments and Dr. J. C. Gentles, of the Department of Medical Mycology, University of Glasgow, for examining the fungus.

\section{REFERENCES}

Fallon, R. J. (1961). J. clin. Path., 14, 505.

Report of the Medical Research Council Working Party on PressureSteam Sterilisers (1960). Lancet, 2, 1243. 